\title{
Large scale simulations in the realm of nanooptics
}

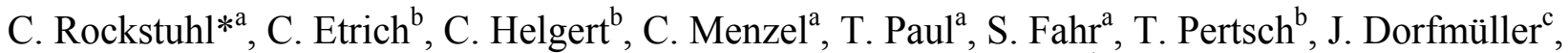

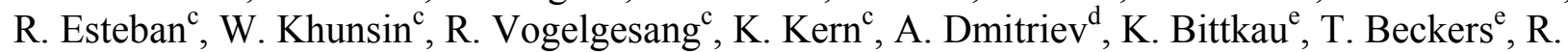 \\ Carius $^{\mathrm{e}}$ and F. Lederer ${ }^{\mathrm{a}}$ \\ ${ }^{a}$ Institut für Festkörpertheorie und -optik, Friedrich-Schiller-Universität Jena, 07743, Jena, Germany \\ ${ }^{\mathrm{b}}$ Institut für Angewandte Physik, Friedrich-Schiller-Universität Jena, 07743 Jena, Germany \\ ${ }^{c}$ Max-Planck-Institut für Festkörperforschung, Heisenbergstr. 1, 70569 Stuttgart, Germany \\ ${ }^{\mathrm{d}}$ Chalmers University of Technology, 41296 Göteborg, Sweden \\ ${ }^{\mathrm{e}}$ Institut für Energieforschung 5, Forschungszentrum Jülich GmbH, 52425 Jülich, Germany
}

\begin{abstract}
The realm of nanooptics is usually characterized by the interaction of light with structures having relevant feature sizes much smaller than the wavelength. To model such problems, a large variety of methods exists. However, most of them either require a periodic arrangement of a unit cell or can handle only single entities. But there exists a great variety of functional devices which may have either a spatial extent much larger than the wavelength and which comprise structural details with sizes in the order of a fraction of the wavelength or they may consist of an amorphous arrangement of strongly scattering entities. Such structures require large scale simulations where the fine details are retained. In this contribution we outline our latest research on such devices and detail the computational peculiarities we have to overcome. Presenting several examples, we show how simulations support the physical understanding of these devices. Examples are randomly textured surfaces used for solar cells, where guided modes excited in the light absorbing layers strongly affect the solar cell efficiency, amorphous metamaterials and stochastically arranged nanoantennas. The usage of computational experiments will be motivated by the unprecedented insight into the functionality of such components.
\end{abstract}

Keywords: Nanooptics, plasmonics, guided waves, metamaterials, solar cells, nanoantennas, near-field optics

\section{INTRODUCTION}

Nanooptics deals with optical effects occurring if light interacts with matter that has artificially structured features with sizes comparable to the wavelength ${ }^{1}$. To induce a sufficiently strong response, different resonance phenomena have to be exploited that are sustained by suitably shaped nanostructures. Three resonant effects may be distinguished.

The first is the excitation of a confined mode either at the interface of a metal and a dielectric (such as propagating surface plasmon polariton), or inside the volume of structured dielectric media (dielectric waveguide mode), or a combination thereof ${ }^{2}$. To excite guided modes by linear optical processes, the momentum mismatch between the free space radiation and the guided modes has to be compensated by an assistive structure ${ }^{3,4}$, since the wave vector for guided modes is required to be outside the light cone $e^{5}$. Such an assistive structure can be either a weakly corrugated modulation of the interface, a shallow dielectric grating, a truncation of the otherwise infinitely extended interface or a strongly scattering isolated element. However, the latter mechanisms are disadvantageous for a resonant excitation, since a coherent build up of light coupled into the waveguide, as it occurs for a periodic arrangement of an assistive structure, cannot be exploited. The second resonance that can be exploited is a Mie resonance ${ }^{6}$. It is excited in isolated nanoparticles made of a dielectric material characterized by a large permittivity. Phenomenologically and in a very simple way, such dielectric nanoparticles are understood as resonators where light may be trapped by means of total internal reflection. Also, light experiences a build up since closed loops exist where the optical path acquires a phase advance by a multiple of $2 \pi$ per round-trip. Naturally, the resonance is never perfect since light leaks out of the structure, causing a broadening and a damping of the resonance. The third resonance that can be exploited is a localized surface plasmon polariton ${ }^{7}$. It is a polaritonic excitation where the charge density oscillation in metallic nanoparticles is coupled resonantly to the impinging electromagnetic wave field.

*carsten.rockstuhl@uni-jena.de; phone +49 (0) 3641947 176; fax +49 (0) 3641947 177; www.photonik.uni-jena.de

Integrated Optics: Devices, Materials, and Technologies XIV, edited by Jean-Emmanuel Broquin, Christoph M. Greiner, Proc. of SPIE Vol. 7604, 76040D · C 2010 SPIE · CCC code: 0277-786X/10/\$18 · doi: 10.1117/12.841700 
In modern nanooptical devices all these effects may occur simultaneously and, except for some distinctive geometries, their appearance cannot be unambiguously separated. To unravel the impact of the different resonances on the optical performance, systematic simulations of the optical devices are often in need.

To reveal the underlying physics of nanooptical devices, today's numerical techniques constitute inevitable tools. First, they allow for a systematic design of optical devices without the need for extensive, resources-consuming experiments. Second, they allow for obtaining insight into the functionality of optical devices which otherwise would be not accessible by any means. And third, they are often necessary to interpret the experimental results obtained with techniques adapted to the world of nanooptics. Such a technique is, e.g., scanning near-field optical microscopy. In the past for all these purposes various numerical techniques were developed to work efficiently in this field. They all aim at rigorously solving Maxwell's equations taking the appropriate constitutive relations fully into account. These methods can be divided roughly into three categories. The first type of numerical techniques makes explicit use of a possible periodic arrangement of the nanostructure and solves Maxwell's equations in the spatial Fourier domain. This is advantageous in the study of gratings, photonic crystals or metamaterials where the unit cells are periodically arranged. Methods like the Fourier Modal Method ${ }^{8}$, the Rigorous Coupled Wave Analysis ${ }^{9}$ or a simple plane wave expansion ${ }^{10}$ to compute the dispersion relation of the eigenmodes in such periodic structures are nowadays indispensable tools. However, their scaling behavior is rather bad and since the associated algorithms cannot be easily parallelized, they remain to be predominantly applicable for computing the optical properties of periodic structures where the periodicity is on the order of the wavelength or slightly larger. Alternatively, a large variety of methods exist which are employed to analyze predominantly the scattering response of isolated nanooptical elements. Examples are the Boundary Element Method ${ }^{11}$, generalized Mie-Scattering ${ }^{12}$ or the Multiple Multipole Method ${ }^{13}$. However, such methods are similarly restricted in most cases to isolated elements and they often scale poorly when very large to (semi-)infinite structures are combined with finite structures in the subwavelength regime, or if singular geometries are present like corners, edges, contact points, or triple-domain lines. For inhomogeneous media, all of these methods essentially fail.

Such structures seem to be amenable only to an analysis using general purpose techniques that solve Maxwell's equations without requiring a specific geometry or symmetry. In our work we will rely on the Finite-Difference TimeDomain method ${ }^{14}$. It can be evaluated on a structured but also on unstructured grids ${ }^{15}$. The algorithm itself is well developed and can be nicely implemented in a parallel computational environment. Therefore, the present availability of large computer clusters allows investigating physical phenomena and the optics in electromagnetically large structures which are characterized by significant features that are much smaller than the wavelength. In this contribution we will present three systems of that type and outline how large scale simulations may contribute to obtaining insight into the physics of such devices. The systems, we are interested in, are randomly textured surfaces as they are used for the purpose of photon management in thin film solar cells, the optical properties of amorphous metamaterials which are characterized by a random arrangement of the metaatoms that constitute the metamaterials, and by the optical properties of metallic nanoantennas and their interaction. For all of these examples we wish to stress that - although a certain feature dominating the optical properties exists - the absence of periodicity in these samples will likewise affect them. Its impact could not have been revealed if either a numerical method is used that only allows to analyze a periodic arrangement of the structural unit elements or if a method is used which is capable to analyze the properties of the individual constituents only.

\section{OUTLINE OF THE NUMERICAL TECHNIQUE}

To obtain a sufficiently precise insight into the properties of light propagation in a nanostructured medium, Maxwell's equations need to be solved rigorously. In the temporal domain they are ${ }^{1}$ :

$$
\begin{aligned}
& \operatorname{rot} \mathbf{E}(\mathbf{r}, t)=-\mu_{0} \frac{\partial \mathbf{H}(\mathbf{r}, t)}{\partial t}, \quad \varepsilon_{0} \operatorname{div} \mathbf{E}(\mathbf{r}, t)=-\operatorname{div} \mathbf{P}(\mathbf{r}, t), \\
& \operatorname{rot} \mathbf{H}(\mathbf{r}, t)=\mathbf{j}(\mathbf{r}, t)+\frac{\partial \mathbf{P}(\mathbf{r}, t)}{\partial t}+\varepsilon_{0} \frac{\partial \mathbf{E}(\mathbf{r}, t)}{\partial t}, \quad \operatorname{div} \mathbf{H}(\mathbf{r}, t)=0,
\end{aligned}
$$

where $\mathbf{E}(\mathbf{r}, t), \mathbf{H}(\mathbf{r}, t), \mathbf{j}(\mathbf{r}, t)$ and $\mathbf{P}(\mathbf{r}, t)$ are the electric field, the magnetic field, the conduction current and the electrical polarization, respectively, and $\varepsilon_{0}$ and $\mu_{0}$ are the free space permittivity and permeability. The equations are incomplete 
without specifying the relation between the polarization/conduction current and the electric field. Assuming either bound excitations or free electrons, in the time domain they may be conveniently written as oscillator equations for the polarization such as

$$
\frac{\partial^{2} \mathbf{P}(\mathbf{r}, t)}{\partial t^{2}}+\gamma \frac{\partial \mathbf{P}(\mathbf{r}, t)}{\partial t}+\omega_{0}^{2} \mathbf{P}(\mathbf{r}, \mathbf{t})=\varepsilon_{0} f \mathbf{E}(\mathbf{r}, t)
$$

with $\omega_{0}, \gamma$ and $f$ being the resonance frequency of the material system, the damping rate and a phenomenologically introduced oscillator strength, or for the current as

$$
\frac{\partial \mathbf{j}(\mathbf{r}, t)}{\partial \mathbf{t}}+\gamma \mathbf{j}(\mathbf{r}, t)=\varepsilon_{0} \omega_{\mathbf{p}}^{2} \mathbf{E}(\mathbf{r}, t)
$$

with $\omega_{\mathrm{p}}$ being the plasma frequency. Equations (1)-(3) constitute a set of coupled differential equations that have to be solved simultaneously. The free parameters in the oscillator equations are frequently matched such that the induced dispersion in the frequency domain matches sufficiently the desired values. As mentioned above, here the method of choice to solve Maxwell's equations rigorously and without any further approximation is the Finite-Difference TimeDomain (FDTD) method ${ }^{14}$. Equations (1)-(3) are directly discretized in space and time on the so-called Yee-grid. Nowadays, the FDTD method can be considered as fairly standard and versatile also in the optical domain ${ }^{16}$. Most notably, in optics, due to the small wavelength as well as structure sizes, a fine spatial mesh is required, leading to large amounts of computer memory needed, which used to be prohibitively costly and/or impossible to realize in commercial hardware. This was overcome in recent years, since memory is available at low cost and can be implemented in large quantities now. Performance is mostly limited by the memory bandwidth. For larger systems and to lower the computation time the FDTD-method can be parallelized straightforwardly by means of the message passing interface (MPI). This is most commonly used these days. To parallelize an FDTD-code by the much simpler means of OpenMP would require a large and expensive shared-memory architecture, while MPI can be used on comparatively low cost clusters of a certain number of distinctive compute-nodes.

\section{RANDOMLY ROUGH SURFACES IN SOLAR CELLS}

Photon management in solar cells is a field of research in optics, which has gained considerable appeal, since nanooptical components may have a large impact and are actually already in place ${ }^{17}$. Photon management, as we understand it here, comprises the problem of how to increase the share of absorbed light in a solar cell with a given design by means of an assistive structure ${ }^{18}$. This is necessary for solar cells where physical constraints limit the absorption. We will concentrate on thin-film solar cells made of hydrogenated amorphous silicon $(a-\mathrm{Si}: \mathrm{H})$. There, the finite diffusion length of the holes limits the physical thickness of the light absorbing layer to only about $250-300 \mathrm{~nm}$. This is insufficient to absorb all incoming photons ${ }^{19}$. The problem might be lifted to a certain extent by resorting to tandem cell concepts, where next to the $a-\mathrm{Si}: \mathrm{H}$ layer a thin microcrystalline silicon $(\mu \mathrm{c}-\mathrm{Si})$ layer is attached. It absorbs the low energy photons that were not absorbed in the top $a$-Si:H cell and if connected in series, increases the external voltage. Nonetheless, the problem remains. Also in tandem solar cells the efficiency could be significantly elevated if more light was absorbed in the top layer. For the purpose of the photon management various approaches are used such as gratings, plasmonic nanostructures, or photonic crystals. A structure that is already in place and used in commercial devices is that of randomly textured surfaces ${ }^{20}$. Their functionality however is poorly understood and their optimization is based on heuristic approaches. In an $a$-Si:H solar cell a transparent conductive oxide (TCO) is deposited on top of a glass substrate. This $\mathrm{TCO}$ surface is usually exposed to diluted $\mathrm{HCl}$ for a short time to etch craters into the layer, thus forming the randomly textured surface ${ }^{21}$. An example of such a surface is shown in Fig. 1(a). These surfaces are characterized by feature sizes of the order of $1 \mu \mathrm{m}$ having a depth up to $1 \mu \mathrm{m}$ and are stochastically distributed on the surface. In any design process of a solar cell, such significant features have to be considered, and most notably their ability to localize

light and to excite guided modes in the light absorbing layer of the solar cell have to be investigated in depth ${ }^{22}$. For this purpose, a large scale parallel computational environment as the one described in section 2 is perfectly suitable. 

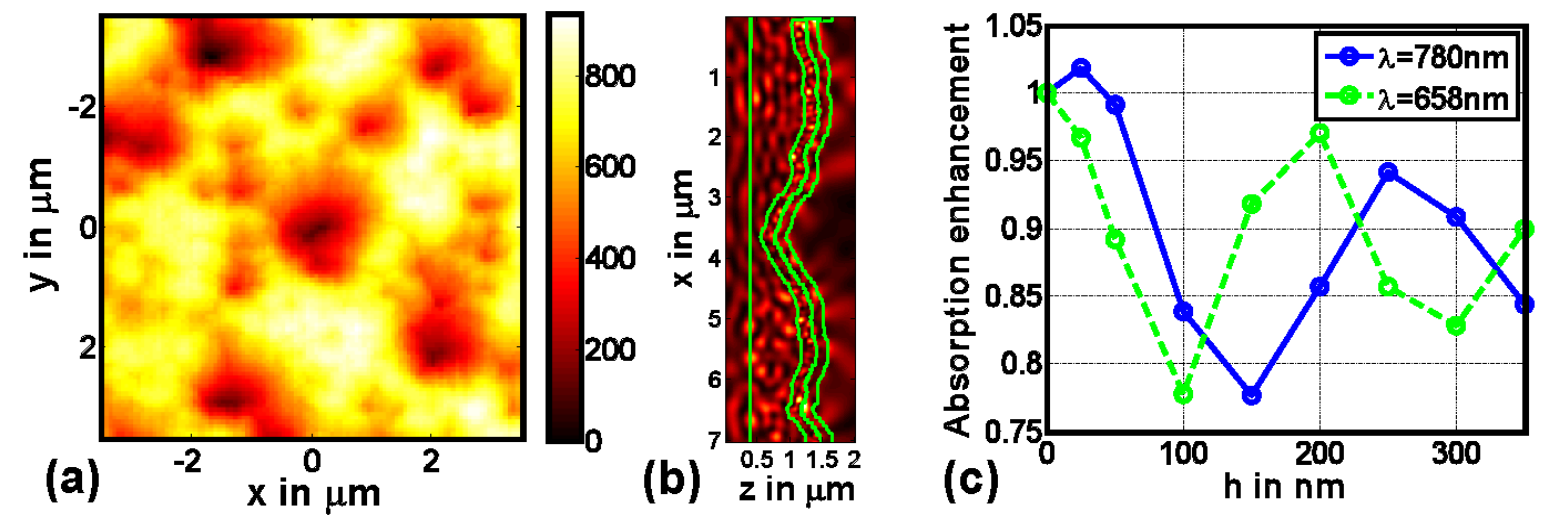

Figure 1. (a) Topography of the random rough surface as considered in this manuscript (height is given in $\mathrm{nm}$ ). (b) Cross section through the device as described in the manuscript with the amplitude of the electric field that evolves upon plane wave illumination. (c) Absorption enhancement in the $a-\mathrm{Si}: \mathrm{H}$ layer of a tandem solar cell as a function of the thickness $h$ of the intermediate layer for two relevant wavelengths [blue solid line denotes $\lambda=780 \mathrm{~nm}$ and green dashed line $\lambda=658 \mathrm{~nm}]$. The cell consists of a randomly textured $\mathrm{ZnO}$ film deposited on a glass substrate followed by a $250 \mathrm{~nm} a$-Si:H layer, the intermediate layer and the substrate.

In our study we take into account surfaces that were measured by an atomic force microscope (AFM) from fabricated devices [see Fig. 1(a)]. We have studied the device with an increasing degree of complexity to reveal the impact of various free parameters that can be modified in a controlled manner ${ }^{23}$. We outline here the results of the dependency of the thickness of an intermediate reflector that is placed between the top and the bottom cell in a tandem solar cell ${ }^{24}$. The cell consists of (1) the glass substrate, (2) the textured transparent conductive oxide (TCO) layer which has a maximum thickness of $1 \mu \mathrm{m}$, (3) the $a$-Si:H layer with a thickness of $250 \mathrm{~nm},(4)$ a $\mathrm{ZnO}$ intermediate layer with a thickness subject to variation and (5) either an infinitely extended half space made of air or made of $\mu \mathrm{c}-\mathrm{Si}: \mathrm{H}$ to mimic the presence of the bottom cell. The infinite extension is a reasonable assumption since its thickness is usually large enough to achieve the state of current matching ${ }^{25}$ required for series connection, i.e., the current generated by the bottom cell equals the current generated by the top cell. Therefore, the necessary thickness is usually such that multiple reflected light from the backside of the cell is negligible in the spectral domain of interest.

To compute the absorption enhancement for a given solar cell, first we calculated the local electromagnetic field everywhere in space using the FDTD method. The surface extending up to $9 \mathrm{x} 9 \mu \mathrm{m}^{2}$ and the thickness of the cell as described above was fully retained. In the longitudinal directions we used perfectly matched layers and perfectly reflecting boundaries to truncate the computational domain; whereas in the transverse directions periodic boundary conditions were imposed. We verified that the absolute size of the spatial domain we considered does not affect the results; hence ensuring that our finite sample sufficiently reflects the stochastic properties of the surface. The sample was illuminated with a linearly polarized plane wave at normal incidence corresponding to different free space wavelengths relevant for the photon management. This spectral domain comprises the wavelength between approx. 600 and $750 \mathrm{~nm}$. It corresponds to the spectral range of the absorption edge. While optimizing the photon management, the primary goal is to shift this absorption edge towards larger wavelengths. The material properties and their dispersion as documented in literature were taken fully into account ${ }^{26}$. Once the electromagnetic field is known, the local dissipation in the $a$-Si:H layer can be extracted by computing the local divergence of the time averaged Poynting vector $\langle\mathbf{S}(\mathbf{r}, \omega)\rangle$,

$$
\operatorname{div}\langle\mathbf{S}(\mathbf{r}, \omega)\rangle=-\frac{1}{2} \operatorname{Im}\left\{\omega \mathbf{P}(\mathbf{r}, \omega) \mathbf{E}^{*}(\mathbf{r}, \omega)\right\}
$$

where $\omega$ is the frequency of the light. Integrating this local absorption over the spatial domain of interest provides a measure for the global absorption. A normalization of this quantity in terms of the global absorption in the solar cell without the assistive structure provides a measure for the absorption enhancement. An example for the computed field distribution in a cross section of a selected device is shown in Fig. 1(b). There, the local magnitude of the electric field is displayed together with lines indicating the boundaries between the different layers. The illumination propagates in the positive z-direction. 

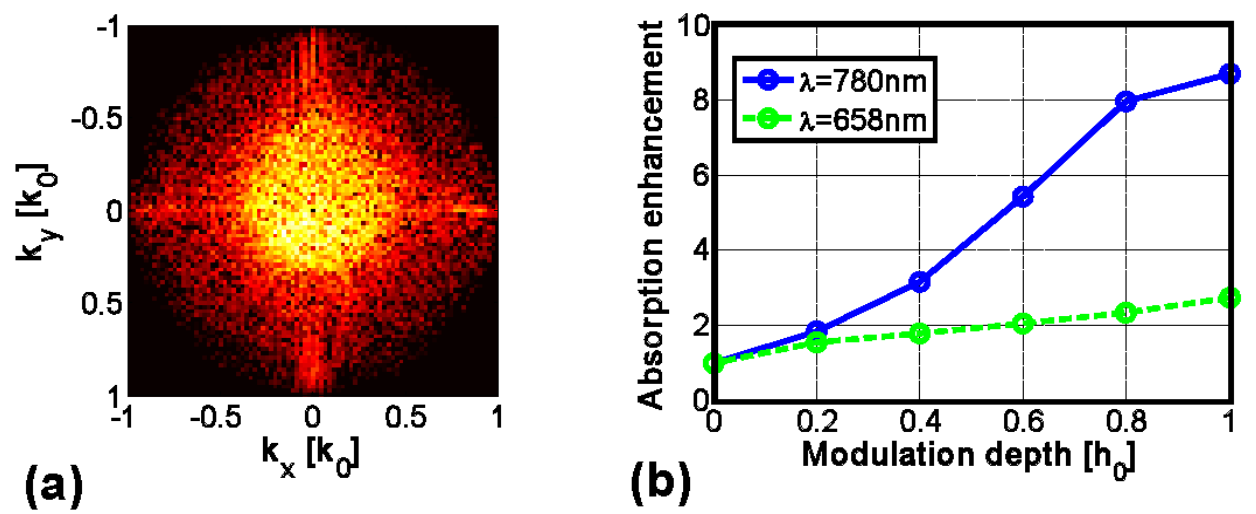

Figure 2. (a) Angularly resolved scattered field (log scale excluding the forward scattered light) by the randomly textured surface into a medium with $n=4$. (b) The absorption enhancement in the $a$-Si:H top cell as a function of the modulation depth of the randomly textured surface for two wavelengths of interest.

Results for the absorption enhancement in the $a-\mathrm{Si}: \mathrm{H}$ solar cell for two representative wavelength as a function of the thickness of the intermediate layer are shown in Fig. 1(c). The backside material in the respective configuration was assumed to be air. Therefore, the predominant impact of the intermediate layer is to decrease the absorption in the $a$ $\mathrm{Si}: \mathrm{H}$, since the layer acts as an anti-reflection coating and mediates the coupling of light out of the cell upon a single passage. However, this deviating feature is not present, of course, in a real device equipped with a bottom cell made of $\mu \mathrm{c}-\mathrm{Si}: \mathrm{H}$. The results from these simulations allow drawing the following conclusions. Most notably, the intermediate layer in such tandem solar cell acts as a Fabry-Perot type cavity where due to multiple reflections inside the layer the amount of reflected light back into the top cell suffers periodic modulations which will spectrally dependent increase or decrease the absorption enhancement. Such behavior is completely in line with expectations from thin film theory and would not justify the undertaken computational effort. However, two subtle details can be found which may have important consequences in the design of such intermediate layers. First, the encountered Fabry-Perot oscillations are not the same as expected for a stratified media with plane interfaces with the same varying thickness. The observed periods are larger by a factor of 1.22 and 1.24 , respectively. This is a result of the intriguing interplay of the light with the randomly textured surface which redirects the light to a large amount from its principle propagation direction. The angularly resolved field scattered by such a surface is shown in Fig. 2(a). This deflection off the principal propagation direction causes the effective propagation constant in the medium to be decreased since only the longitudinal wave vector component is important. This change of the effective propagation constant in the randomly textured material needs to be fully considered in the optimal design of the intermediate layer. Therefore, the optimal thickness for an absorption enhancement will deviate from the value as deduced from considerations that are based on thin film approximations with planar interfaces. The second important aspect is the loss of spatial coherence. Coherence, loosely defined as the ability of the light to interfere, gradually decreases which reduces the modulation depth of the Fabry-Perot oscillations. This is not surprising since light suffering multiple scattering events will no more pulsate with a fixed phase relation but may oscillate statistically out of phase. This implies that for intermediate reflectors being thick enough, any excessive design process that aims to adjust the absorption maxima of the Fabry-Perot oscillation to some predefined wavelength will be pointless, since such interference effects will fade away. For a large thickness of the intermediate layer the remaining absorption enhancement converges towards the value for incoherent light. Its magnitude can be predicted by assuming incoherent reflections at both interfaces of the intermediate layer.

What cannot be seen in Fig. 1(c) is the actual increase of the absorption that occurs due to the randomly textured interface. This can be tremendous, depending on the wavelength. Figure 2(b) shows the absorption enhancement in a top cell of a tandem solar cell as a function of the modulation depth of the randomly textured surface. For long wavelengths, where the absolute absorption in the $a-\mathrm{Si}: \mathrm{H}$ is low, the enhancement tends to be as large as 10; whereas at smaller wavelengths, where the absolute absorption is high, values for the enhancement as large as 3 are attained. Nevertheless, this factor implies that the majority of light is absorbed and the system is close to saturation for this wavelength-the monochromatic absorption obviously cannot exceed $100 \%$. (The spectrally integrated absorption is bound to even lower limits due to material and thermodynamic considerations like the famous Shockley-Queisser limit of 30\% for single p-n junctions in $\mathrm{Si}$.) 
Overall, from these considerations it turns out that large scale simulations are a versatile tool to deduce physical functionalities in such devices as well as to perform design tasks with the ultimate goal to maximize the absorption in the solar cell.

\section{AMORPHOUS METAMATERIALS}

A second field of research where large scale simulations are useful for revealing new physical aspects are amorphous metamaterials. Metamaterials are made of strongly scattering units, commonly called metaatoms, which are so small that the propagating light will not resolve the spatial details of the structure but merely experiences an effective medium ${ }^{27}$. Such media may exhibit optical properties not available in nature. In most cases at the focus of interest is the ability of the metamaterial to show the effect of negative refraction ${ }^{28}$. Also an effective permittivity much larger than that available in any natural material is a remarkable property ${ }^{29}$. Having such materials readily at hand allows implementing unprecedented applications such as, e.g., a perfect lens or a cloak ${ }^{30}$. For ease of fabrication, and, even more important, to simplify the design and the numerical analysis of such artificial materials, the metaatoms are arranged usually periodically. However, a periodic analysis and the fabrication of such materials are restrictive for the following reasons. First, the periodic arrangement stipulates undesired properties associated with the excitation of higher order Bloch modes such as, e.g., anti-resonances in the effective properties. Second, the strong interaction among metaatoms when they are spaced in close spatial proximity, promotes their coupling, leading to undesired resonances which should be suppressed. Third, a dilution in excess to avoid the two problems above would also lower the excitation strength and thus the possibly induced dispersion in the effective properties. Fourth, since most of the metaatoms of current interest have an anisotropic polarizability, their arrangement in a periodic lattice also leads to anisotropic effective properties. This is undesirable for a large fraction of applications which were envisioned having isotropic materials at hand. Such limitation could be lifted by having an amorphous arrangement of unit cells in space. Fifth, and arguably most important, the currently used top-down approaches for the fabrication of metamaterials are not promising for obtaining true bulk quantities. Instead bottom-up approaches using self-organization of suitably tailored metaatoms in three dimensions should be exploited. For all these reasons the property of non-periodically arranged unit cells should be explored ${ }^{31}$ which necessarily requires the use of large scale computational facilities ${ }^{32}$. In the simulations the very fine details of the metaatoms, and at the same time a sufficiently large number to reflect the amorphous character, should be properly taken into account.
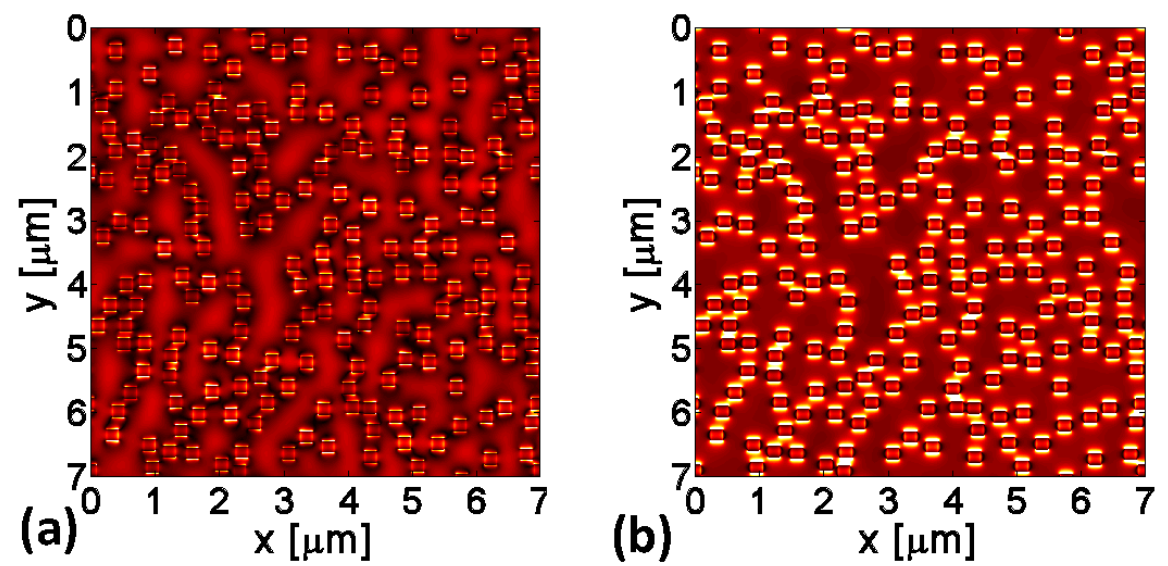

Figure 3. Amplitude distribution of the y-polarized electric field $10 \mathrm{~nm}$ above a single layer of metaatoms in an amorphous arrangement illuminated with a $y$-polarized plane wave at normal incidence with a wavelength of $797 \mathrm{~nm}$ in (a) and with a wavelength of $1094 \mathrm{~nm}$ in (b). The same linear color scale is used in both panels.

In the current work we investigated exemplarily the impact of a random arrangement of a prototypical metaatom, namely the double cut-wire structure, on the observable spectra and the effective properties. The double cut-wire has a symmetrical cross section in the transversal direction of $180 \mathrm{x} 180 \mathrm{~nm}^{2}$. The $C_{4}$ symmetry was chosen since it supports a polarization independent response. The double cut-wires were made of a sequence of Au-MgO-Au where the layers had a thickness of $30 \mathrm{~nm}, 45 \mathrm{~nm}$, and $30 \mathrm{~nm}$, respectively. The referential unit cell was assumed to have a period of $512 \mathrm{~nm}$ in each direction. The amorphous arrangement was generated by displacing each metaatom from the center of its unit cell 
randomly by $\Delta x \in\left[-\frac{D \Lambda_{x}}{2}, \frac{D \Lambda_{x}}{2}\right]$ and $\Delta y \in\left[-\frac{D \Lambda_{y}}{2}, \frac{D \Lambda_{y}}{2}\right]$,where $\Delta x$ and $\Delta y$ were uniformly distributed.

Here, $D$ is a dimensionless parameter which was chosen to be 3 to allow for a complete randomization. In addition we have enforced the particles to have a minimal distance to avoid a too strong coupling. In the simulations we took a super cell of $7 \times 7 \mu \mathrm{m}^{2}$ into account and imposed periodic boundary conditions to keep the system numerically tractable. This allows maintaining overall 196 metaatoms in the transversal plane in the simulations. This sufficiently large number assures that the results will not depend too much on the characteristics of an individual implementation of the randomness. Simulations here are only shown for structures where a random displacement is enforced for a single layer. In the FDTD simulations the space was discretized using a resolution of $5 \mathrm{~nm}$. Each wavelength was simulated separately to allow incorporating the real material parameters in an easy way. For the metallic response the Drude model was used to simulate the induced current. Here the plasma frequency and the damping constant were adjusted. The use of additional oscillators as defined in Eq. (2) to model the metallic response of an incoming pulse correctly may lead to excessive memory requirements given the size of the geometry. The entire system was truncated in the propagation direction by perfectly matched layers and it was illuminated with a linearly polarized plane wave. The temporal evolution of the system was computed sufficiently long to obtain the steady-state field. The amplitude of the excited component of the electric field in a cross section above the structure at two wavelengths where resonances are sustained by the system is shown in Fig. 3.
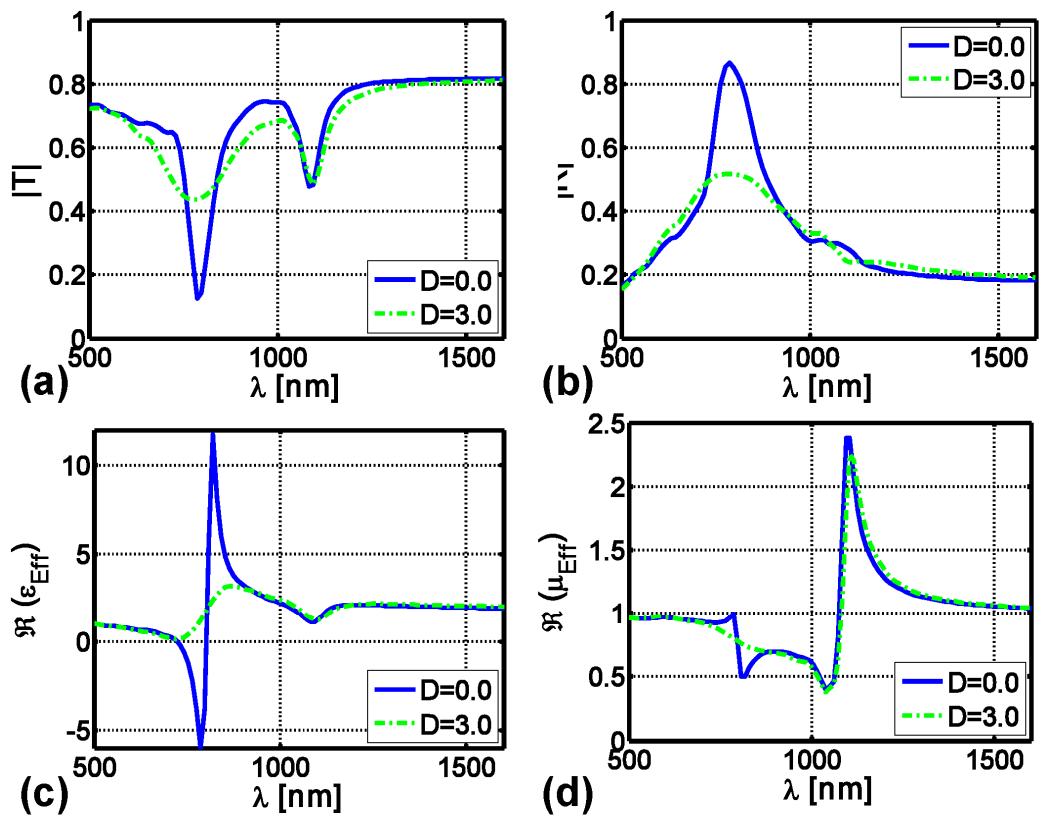

Figure 4. Transmission (a) and reflection (b) spectra of the metamaterial for a periodic arrangement $(D=0$, blue solid line) and an amorphous arrangement $(D=3$, green dashed line). The real part of the effective properties are shown as well and resolved in the permittivity (c) and the permeability (d).

From such transmitted locally resolved amplitudes the complex transmission can be extracted by imposing a spatial Fourier-transformation of the complex field. An equivalent procedure is applied to the reflected field whereas care has to be taken while subtracting the incident field from the total field to deduce the share of reflected light. The complex reflection and transmission can be used to retrieve effective properties ${ }^{35}$. They are, as usual, not understood in the rigorous sense of being material properties but only as properties assigned to an isotropic homogenous medium replacing the nanostructured layer which causes the same optical coefficients as the nanostructured material. Spectrally resolved transmitted and reflected light is shown in Fig. 4 (a) and (b). Results are shown for two different values of the parameter $D$, where $D=0$ corresponds to the perfect periodic case. For values of $D$ larger than unity the structure can be understood as fully amorphous since the statistical displacement of the unit cell from its regular grid position, which exceeds (on average) the size of one unit cell, and the actual magnitude of $D$ are not important anymore. This can be deduced also from supplementary simulations. Physically, the spectra show two resonances, appearing as dips in 
transmission or peaks in reflection. They are understood as the symmetric and anti-symmetric localized plasmon polariton excited in the two cut-wires. In the transition from the periodic to the amorphous arrangement the resonance wavelengths are not affected, as it is anticipated for a system that supports resonances in its individual elements. However, the line widths of both resonances are affected differently. The resonance of the symmetric plasmon mode at the smaller wavelength is significantly weakened and damped while the resonance of the anti-symmetric plasmon mode at the larger wavelength survives the transition to disorder of arbitrary degree.

The same trend is also reflected in the effective properties as shown in Fig. 4 (c) and (d). Please note that for brevity only the real parts of the effective properties are shown for the two scenarios. Comparing the periodic and the amorphous arrangement, it can be seen that the induced dispersion in the permeability at the longer wavelength is not affected at all and remains insensitive against disorder. In contrast, the induced dispersion in the permeability at the shorter wavelength, being reminiscent of an anti-Lorentzian shape and usually regarded as an artifact of periodic arrangements, strongly fades away and only a smooth curve persists. At shorter wavelengths, the dispersion in the permittivity remains noticeable, though at a much weaker level. This is in full agreement with the spectral observations. Similarly, we can conclude here that the resonance tends to be weakened and damped the stronger the disorder is in the lattice.

This feature is quite different for both wavelengths. It may be explained with the peculiarities of the scattering response of the individual metaatom. For the symmetric mode the scattered field is dominated by an electric dipole (inducing the dispersion of the permittivity) having a strong in-plane polarized component in the plane of the metamaterial. This scattered field interferes coherently with the illuminating field and drives each metaatom slightly out of phase. The loss of coherence leads to a damping of the resonance which is homogenously broadened. In contrast, for the anti-symmetric long wavelength resonance the scattered field of the metaatom is dominated by an electric quadrupole, which leads to a dispersive permeability in the limit of weak spatial dispersion ${ }^{36}$. The electric quadrupole however, does not have an inplane field component in the plane of the metaatoms. No secondary wave field can affect the illumination and this suppresses any further interparticle coupling at this particular resonance. This is quite intriguing and may allow to judge on the character of the resonance of a certain metaatom without applying a sophisticated retrieval procedure.

The main conclusions that could be revealed by such large scale simulations are the following. First, the resonances that induce any dispersion in the effective properties remain excitable even if the metaatoms are not placed on a periodic grid. Second, the transition to an amorphous medium only causes a weakening and a broadening of the resonance; predominantly for the electric dipolar modes. Only such eigenmodes have a strong field component in the plane of interest which might affect the scattering response of neighboring metaatoms. All these results are very promising to open the path towards amorphous metamaterials made of a high density of metaatoms which are arranged using self organized bottom-up techniques.

\section{OPTICAL NANOANTENNAS AND THEIR COUPLING}

The last field of research, for which large scale simulations provided unprecedented insight, is quantitative near-field microscopy. A scanning near-field optical microscope (SNOM) is generally capable of picking up the field locally in spatial proximity to an object of interest by a suitably tailored tip ${ }^{33}$. If part of the evanescent field around a nanoobject is converted by means of the instrument into propagating waves, the resolution can be well beyond that of classical farfield optics. Various implementations for a SNOM exist, although we concentrate here on a so-called scattering type, apertureless SNOM (aSNOM) ${ }^{34}$. Its advantage is the potential spatial resolution that is in the order of the tip radius. Often a limiting aspect of aSNOM, and all other instrumental SNOM implementations as well, is the disturbance of the near-field by a probing tip required in order to couple the evanescent field into far-field modes. If this process is accompanied by significant back-coupling from the tip into the observed nearfield modes, the very field is altered by the observing instrument actually that it is intended to measure. This renders the interpretation of the measured images often cumbersome or impossible without a priori knowledge. Indeed, frequently SNOM is employed to observe a specific field distribution rather than its quantitative observation free of any assumptions ${ }^{37}$. For these reasons computational approaches are an indispensable tool to compare measurements with simulations and to actually allow for their interpretation. Moreover, for doing quantitative near-field optical microscopy a large number of samples need to be measured simultaneously with the same instrument in an identical state of adjustment to exclude any deviations that may occur due to the irreproducibility of the measurement ${ }^{40}$. With suitable data recorded, simulations of these large area structures comprising many samples crucially help to decide whether a specific measured field is reminiscent of the very local details of the structure or, whether it is depending on the SNOM system and may be rather affected by the imperfections of the measurements. 
Figure 5 shows a representative set of measurement (a) and simulation (b). The sample under investigation is comprised of 20-nm-thick metallic nanodiscs with two different diameters: either $216 \mathrm{~nm}$ or $350 \mathrm{~nm}^{39}$. They were fabricated bottomup with a self-organization approach. There, dielectric beads were dispensed at a surface of a dielectric substrate ${ }^{38}$. Using a shadow lithographic technique and suitable evaporation methods allow for their transformation to small metallic nanodiscs. Since the beads were charged during their deposition, they tend to arrange themselves with a maximal possible distance, ensuring minimal interaction. The size of the nanodiscs was chosen intentionally. Whereas the small nanodiscs should support a dipolar resonance at the measurement wavelength of $875 \mathrm{~nm}$, the large nanodiscs have a sufficient size to sustain a higher order plasmonic eigenmode; namely a quadrupolar resonance. It should be noted that this quadrupolar resonance cannot be excited at normal incidence due to symmetry constraints but requires oblique incidence. The measurements were done with an aSNOM as described in Ref. 41. The sample was illuminated with a weakly focused Gaussian beam with a nominal incidence angle of $70^{\circ}$ relative to the normal of the sample. With respect to the incidence plane the field was TE-polarized; i.e., the electric field was polarized perpendicular to the incidence plane. With this polarization the electric field is able to excite the above mentioned plasmonic eigenmodes. It was stipulated that the field component $\mathrm{E}_{\mathrm{z}}$ normal to the surface was detected since the aSNOM tip (made of Si without any metallic coating) exhibits the strongest polarizability along this direction. Hence, only this component is picked-up by the tip and re-scattered into the far-field. Lock-in techniques and a heterodyne scheme allow recording both amplitude and phase of the scattered field simultaneously.
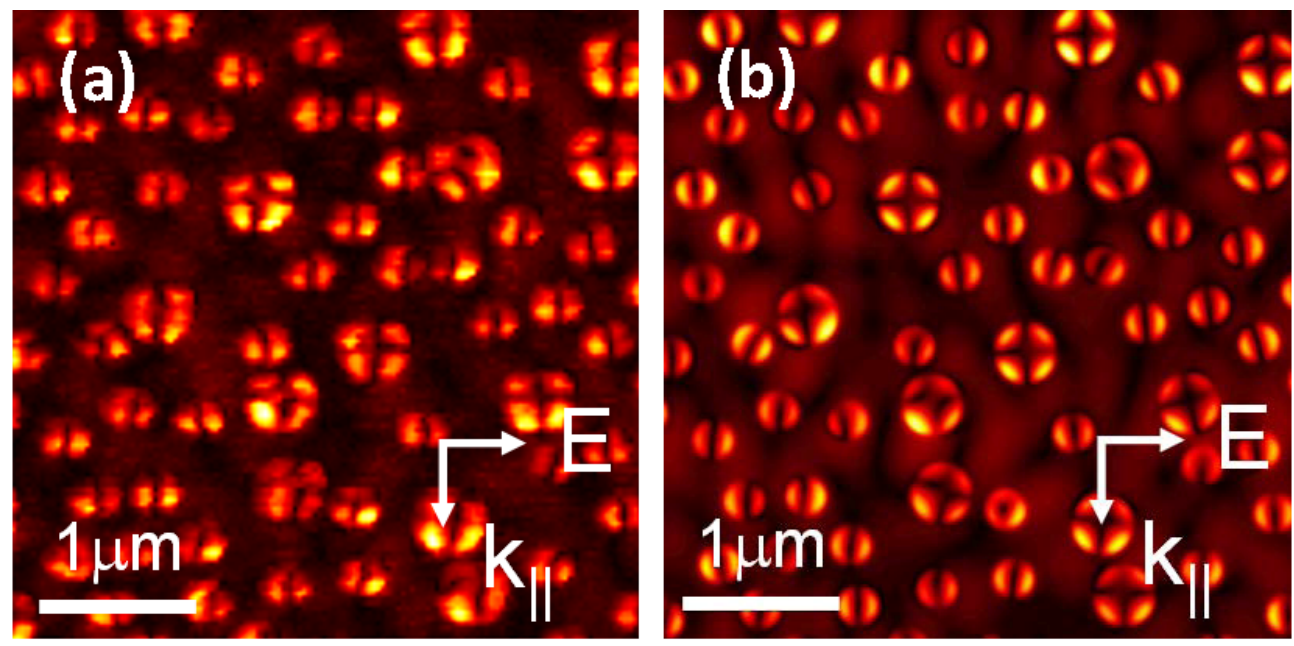

Figure 5. Measured (a) and simulated (b) distribution of the absolute value of the electric field component normal to the surface of a sample comprised of nanoantennas of two different sizes which sustain either a dipolar resonance or a quadrupolar resonance. Illumination conditions are indicated in the figure.

The simulations were done by extracting the spatial position of the nanodiscs from AFM images recorded simultaneously with the aSNOM images. In a first approach we used the nominal size of the nanodiscs for the simulations and not the size as it could be deduced from the AFM measurements. This turned out to be sufficient to allow for a full reconstruction of all experimental images. The size of the sample considered in the simulations was $5 \times 5 \mu \mathrm{m}^{2}$, comprising $\sim 40$ small and $\sim 15$ large nanodiscs. As in the simulations before, we imposed periodic boundaries in the transverse plane, i.e., the plane of the sample, and perfectly matched layers along the surface-normal direction. Deviating from the experimental set-up, we assumed an illumination with a plane and not with a gently focused Gaussian wave. This however, does not affect the results since the gently focused Gaussian beam is locally sufficiently approximated by a plane wave which is assumed to excite the plasmonic resonances. This is justified since the size of the nanodiscs is smaller than the wavelength and much smaller than the waist of the focused Gaussian beam. To allow for an easy comparison between measurement and simulation, Fig. 5 (b) shows also only the electric field component normal to the sample. It has to be stressed that this component is not included in the illumination but originates exclusively from the scattered field of the nanodiscs. The spatial resolution in the simulation was $4 \mathrm{~nm}$ and a Courant factor of 2 was imposed.

From the measured results alone, as shown in Fig. 5 (a), it can be seen that the sample sustains indeed two different kinds of plasmonic eigenmodes. The dipolar mode is characterized by two amplitude lobes in the $z$-component, whereas the quadrupolar mode sustains four lobes. They are unambiguously resolved and their spatial position can be uniquely correlated to the spatial position of the small and the large nanodiscs, respectively. Therefore, it can be concluded that 
the principal field patterns measured are not affected by artifacts. Nevertheless, without quantitative numerical comparison, the measured figures seem discouraging at first glance since the fields around the nanodiscs are not identical, despite their presumably identical shape. They show deviating features from nanodisc to nanodisc. Most notably the orientations of the dipoles and quadrupoles from nanodisc to nanodisc appear slightly rotated and show a deviation from their pivotal orientation perpendicular to the in-plane component of the illuminating wave vector. This suggests at first sight that local deviations in the geometry among the nanodiscs affect the results and prevents therefore a unique interpretation.

However, this turned out to be misleading, since the large scale FDTD simulations could uniquely reproduce the measured field distributions. Very fine details were fully resolved. In a supplementary simulation (not shown here for brevity) we simulated the near field of individual small and large nanodiscs and displaced these fields over the sample to spatial positions where a nanodisc of the respective size is located. The mere coherent superposition of these fields was insufficient to describe the re-orientation and re-shaping of the fields around the nanodiscs. This strongly suggests that secondary scattering processes from the nanodiscs are responsible for the unique near-field patterns that are finally observed. According to this image, the scattered field from a certain nanodisc has an in-plane component which may induce a scattered field in all the other nanodiscs in the sample. Please note that the scattered field has a normal component as well; but since the particles are non-resonant for such a polarization it is not expected that any significant share of a secondary field is induced. The field that is finally observed in the vicinity to a certain nanodisc is a superposition of the primary scattered field (as induced by the illumination) and the secondary scattered field (as induced by the scattered field of all the other nanodiscs). Since this secondary field may have an $x$ - and a $y$-component, the overall eigenmode appears to be rotated. It is worth mentioning that predominantly nearest neighbors induce a secondary scattered field since the magnitude of the scattered field scales with $r^{-3}$ for the dipole and $r^{-4}$ for the quadrupole. Nevertheless, this different scaling behavior of the scattered field might affect the magnitude of the modifications of the primary scattered field. In consequence, the dipolar fields appear much stronger than the quadrupolar fields. More quantitative statements are beyond what is seemingly possible with such an interpretation.

All these insights would have been inaccessible if not large scale simulations were conducted. The simulation of an isolated element is insufficient since, obviously, the overall field distribution is dictated by the coherent superposition of a large number of mutual scattering interactions. On the other hand, simulations of a periodic array would not have made it possible to understand the measured field patterns since then the fields of neighboring particles would interfere constructively with a fixed phase relation. The breakup of this fixed phase relation by a random arrangement of the nanodiscs made it possible to explain the very fine details that were observed. Moreover, and this aspect has to be stressed, it was possible to show that the very subtle details of the field patterns that were measured are not caused by deviations and imperfections in the geometries of the individual elements or detrimental feedback interactions induced by the probe tip. Instead, they are due to a coherent interaction of the ensemble. This analysis provide strong evidence that the measured field distributions with the aSNOM are correct in a sense that they correspond to the component of the local field that is normal to the surface in the absence of the tip.

\section{CONCLUSIONS}

In conclusion, we detailed for three examples how the use of large scale computational efforts allows obtaining insight into physical systems which are characterized by nanometric objects dispersed over surfaces significantly surpassing one wavelength in extent. Moreover, this random or amorphous arrangement induces peculiarities in the process of light propagation which could not have been anticipated from considerations of the periodic arrangement or from the simulations of isolated elements. It has to be stressed that simulation techniques such as the outlined Finite-Difference Time-Domain method are perfectly suited for this kind of problem since they scale linearly with size, can be easily extended to run simultaneously on a large number of processors and have negligible overhead, hence it also scales in sufficiently good approximation linearly with the size of the cluster.

The three examples for application of large scale optical simulations were the field of photon management for solar cells, metamaterials and optical nanoantennas, although other fields are easily imaginable, such as optics in high-Q microresonators, complex photonic crystal circuits, quasi-periodic photonic structures, or the wide field of optical clusters. In all these scenarios analytical considerations may predict optical quantities of interest but often simulations are required for quantitative predictions. A certain limiting factor in all the examples, also including these discussed in the present work, is the persistent difficulty to routinely conduct a series of simulations on the optical properties of the respective systems. This is desirable if, e.g., it is aimed to spectrally resolve the ability of a randomly textured surface to enhance 
the absorption in a thin film solar cell. Simulations for some isolated wavelengths are useful to generate a principle understanding of the system, but the solar cell is not a device which is required to operate in an optimal way in a certain spectral interval. It rather requires some broad band optimization. Moreover, for other fields not outlined in the present work, a parameter scan of some geometrical details of the structure to optimize certain functionalities, such as, e.g., a figure of merit for a negative index material would also be beyond what is currently possible in a realistic time frame, unless access to a computer cluster comprising sufficiently many nodes is provided.

\section{ACKNOWLEDGEMENTS}

The authors acknowledge support by the Federal Ministry of Education and Research (Unternehmen Region, ZIK ultra optics, Metamat, PhoNa, Nanovolt), the German Science Foundation (Nanosun PAK88) and the European Union (Nanogold). Some computations utilized the IBM p690 cluster JUMP of the Forschungszentrum in Jülich, Germany.

\section{REFERENCES}

[1] Novotny, L. and Hecht, B., [Principles of Nano-Optics] Cambridge University Press, (2006).

[2] Love, J. and Snyder, A. W., [Optical Waveguide Theory] Springer Press, (1983).

[3] Otto, A., "Excitation of nonradiative surface plasma waves in silver by the method of frustrated total reflection," Zeitschrift für Physik 216, 398-410 (1968).

[4] Kretschmann, E. and Raether, H., "Radiative decay of nonradiative surface plasmons excited by light," Z. Naturforsch. 23A, 2135-2136 (1968).

[5] Joannopoulos, J. D., Johnson, S. G., and Winn, J. N., [Photonic Crystals: Molding the Flow of Light] Princeton University Press, (2008).

[6] van De Hulst, H. C., [Light Scattering by Small Particles] Dover Publication Inc., (1982).

[7] Maier, S. A., [Plasmonics: Fundamentals and Applications] Springer, Berlin, (2007).

[8] Li, L., "New formulation of the Fourier modal method for crossed surface-relief gratings," J. Opt. Soc. Am. A 14, 2758-2767 (1997).

[9] Moharam, M. G., Grann, E. B., Pommet, D. A., and Gaylord, T. K. , "Formulation for stable and efficient implementation of the rigorous coupled-wave analysis of binary gratings," J. Opt. Soc. Am. A 12, 1068-1076 (1995).

[10] Rockstuhl, C., Menzel, C., Paul, T., Pertsch, T., and Lederer, F., "Light propagation in a fishnet metamaterial", Phys. Rev. B 78, 155102, (2008).

[11] Rockstuhl, C., Salt, M. G., and Herzig, H. P., "Analyzing the scattering properties of coupled metallic nanoparticles", J. Opt. Soc. Am. A 21, 1761-1768 (2004).

[12] Wriedt T. "A review of elastic light scattering theories," Part. Part. Syst. Charact. 5, 67-74 (1998).

[13] Hafner, C., [The Generalized Multipole Technique for Computational Electromagnetics] Artech House Inc. (1990).

[14] Taflove, A. and Hagness, S. C., [Computational Electrodynamics: The Finite-Difference Time-Domain Method] Artech House Inc. (2005).

[15] Niegemann J., König M., Stannigel K. and Busch K. "Higher-order time-domain methods for the analysis of nanophotonic systems," Photonics Nanostruct. 7, 2-11 (2009).

[16] Farjadpour, A., Roundy, D., Rodriguez, A., Ibanescu, M., Bermel, P., Joannopoulos, J. D., Johnson, S. G., and Burr, G. "Improving accuracy by subpixel smoothing in FDTD," Opt. Lett. 31, 2972-2974 (2006).

[17] Würfel, P., [Physics of Solar Cells: From Basic Principles to Advanced Concepts] Wiley-VCH (2009).

[18]Ulbrich, C., Fahr, S., Üpping, J., Peters, M., Kirchartz, T., Rockstuhl, C., Wehrspohn, R., Gombert, A., Lederer, F., and Rau, U., "Ultra-light-trapping and directional selectivity in solar cells", Phys. Stat. Sol. (a) 205 2831-2843 (2008).

[19] Carlson, D.E., and Wronski, C.R., "Amorphous silicon solar cell," Appl. Phys. Lett. 28, 671-673 (1976).

[20] Yablonovitch, E., "Statistical ray optics," J. Opt. Soc. Am. 72, 899-907 (1982).

[21] Bittkau, K., Beckers, T., Fahr, S., Rockstuhl, C., Lederer, F., and Carius, R., "Nanoscale investigation of lighttrapping in a-Si:H solar cell structures with randomly textured interfaces, " Phys. Stat. Sol. (a) 205, 2766-2776, (2008)

[22] Fahr, S., Rockstuhl, C., and Lederer, F., "Engineering the randomness for enhanced absorption in solar cells," Appl. Phys. Lett. 92, 171114, (2008).

[23] Rockstuhl, C., Fahr, S., Lederer, F., Beckers, T., Bittkau, K., and Carius, R., "Local versus global absorption in thinfilm solar cells with randomly textured surfaces," Appl. Phys. Lett. 93, 061105 (2008). 
[24] Rockstuhl, C., Bittkau, K., Beckers, T., Carius, R., and Lederer, F., "The impact of intermediate reflectors on light absorption in tandem solar cells with randomly textured surfaces," Appl. Phys. Lett. 94, 211101, (2009).

[25] Bielawny, A., Rockstuhl, C., Lederer, F., and Wehrspohn, R. B., "Intermediate reflectors for enhanced top cell performance in photovoltaic tandem cells," Opt. Express 17, 8439-8446, (2009).

[26] Vetterl, O., Finger, F., Carius, R., Hapke, P., Houben, L., Kluth, O., Lambertz, A., Mück, A., Rech, B., and Wagner, H., "Intrinsic microcrystalline silicon: A new material for photovoltaics, " Sol. Energy Mater. Sol. Cells 62, 97-108 (2000).

[27] Engheta, N. and Ziolkowski, R. W., [Electromagnetic Metamaterials: Physics and Engineering Aspects] Wiley \& Sons (2006).

[28] Paul, T., Rockstuhl, C., Menzel, C., and Lederer, F., "Anomalous refraction, diffraction and imaging in metamaterials, " Phys. Rev. B 79, 115430 (2009).

[29] Rockstuhl, C. and Scharf, T., "A metamaterial based on coupled metallic nanoparticles and its band-gap property", Journal of Microscopy 229, 281-286, (2008).

[30] Valentine, J., Li, J., Zentgraf, T., Bartal, G., and Zhang. X., "An optical cloak made of dielectrics," Nature Materials 8, 568-571, (2009).

[31] Papasimakis, N., Fedotov, V. A., Fu, Y. H., Tsai, D. P., and Zheludev, N. I. , "Coherent and incoherent metamaterials and order-disorder transitions", Phys. Rev. B 80, 041102(R) (2009).

[32] Helgert, C., Rockstuhl, C., Etrich, C., Menzel, C., Kley, E.-B., Tünnermann, A., Lederer, F., and Pertsch, T., "Effective properties of amorphous metamaterials", Phys. Rev. B 79, 233107 (2009).

[33] Smith, D. R., Schultz, S., Markoš, P., and Soukoulis, C. M., "Determination of effective permittivity and permeability of metamaterials from reflection and transmission coefficients," Phys. Rev. A 78, 043811 (2008).

[34] Petschulat, J., Chipouline, A., Pertsch, T., Tünnermann, A., Rockstuhl, C., Menzel, C., and Lederer, F., "On the dispersion relation in metamaterials - an analytical approach", Phys. Rev. A 78, 043811 (2008).

[35] Ash, E. A. and Nicholls, G., "Super-resolution Aperture Scanning Microscope," Nature 237, 510-512 (1972).

[36]F. Zenhausern, M. P. O’Boyle, and H. K. Wickramasinghe, "Apertureless near-field optical microscope", Appl. Phys. Lett. 65 (13), 1623-1625 (1994) and F. Zennhausern, Y. Martin, and H. K. Wickramasinghe, "Scanning Interferometric Apertureless Microscopy: Optical Imaging at 10 Angstrom Resolution", SCIENCE, 269, 1083-1085 (1995)..

[37] Hecht, B., Bielefeldt, H., Inouye, Y., Pohl, D. W., and Novotny, L., "Facts and artifacts in near-field optical microscopy," J. Appl. Phys. 84, 2492-2498 (1997).

[38] Dorfmüller, J., Vogelgesang, R., Weitz, R. T., Rockstuhl, C., Etrich, C., Pertsch, T., Lederer, F., and Kern, K., "The Fabry-Pérot resonances in one-dimensional plasmonic nano-antennas," Nano Letters 9, 2372-2377 (2009).

[39] Esteban, R., Vogelgesang, R., Dorfmüller, J., Dmitriev, A., Rockstuhl, C., Etrich, C., and Kern, K., "Direct nearfield optical observation of higher order plasmonic resonances, " Nano Letters 8, 3155-3159 (2008).

[40] Fredriksson, H., Alaverdyan, Y., Dmitriev, A., Langhammer, C., Sutherland, D. S., Zäch, M., Kasemo, B., "HoleMask Colloidal Lithography," Advanced. Mater. 19, 4297-4302 (2007).

[41]Bek, A., Vogelgesang, R., and Kern, K., "Apertureless scanning near field optical microscope with sub-10 nm resolution," Rev. Sci. Instrum. 77, 043703 (2006). 